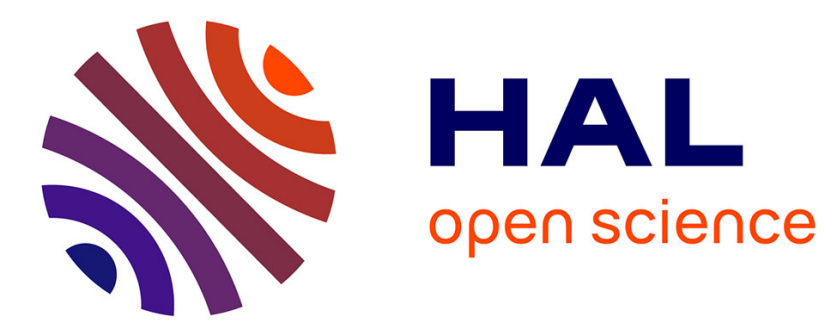

\title{
Intégration des caractéristiques des soudures dans les calculs de crash
}

\author{
P. Huss, M. Dannawi, S. Marya
}

\section{To cite this version:}

P. Huss, M. Dannawi, S. Marya. Intégration des caractéristiques des soudures dans les calculs de crash. Journal de Physique IV Proceedings, 1997, 07 (C3), pp.C3-957-C3-962. 10.1051/jp4:19973161 . jpa00255451

\section{HAL Id: jpa-00255451 https://hal.science/jpa-00255451}

Submitted on 1 Jan 1997

HAL is a multi-disciplinary open access archive for the deposit and dissemination of scientific research documents, whether they are published or not. The documents may come from teaching and research institutions in France or abroad, or from public or private research centers.
L'archive ouverte pluridisciplinaire HAL, est destinée au dépôt et à la diffusion de documents scientifiques de niveau recherche, publiés ou non, émanant des établissements d'enseignement et de recherche français ou étrangers, des laboratoires publics ou privés. 


\title{
Intégration des caractéristiques des soudures dans les calculs de crash
}

\author{
P. Huss, M. Dannawi et S. Marya \\ Laboratoire Mécanique et Matériaux, École Centrale de Nantes, 1 nue de la Noé, B.P. 92101, \\ 44321 Nantes cedex 03, France
}

\begin{abstract}
Résumé. Cet article s'inscrit dans le cadre d'une tentative d'amélioration des simujations numériques de structures mécano-soudés. Plusieurs chemins de modélisation d'une structure sont proposés en intégrant les éléments simulant la soudure. Ainsi complété, le modèle numérique devient plus physique et, par la même occasion, moins sensible aux paramètres numériques et conditions aux limites. Les déformées initiales dues au soudage sont simulées pour évaluer la sensibilité du modèle aux imperfections géométriques.
\end{abstract}

\begin{abstract}
This article describes an attempt to improve the numerical simulation of welded structures. Several ways of modeling a structure are proposed by integrating finite elements simulating welding beads. Hence, the FE model becomes more physical and less sensitive to numerical parameters and boundary conditions. Initial unevenness induced by welding processes is simulated so as to assess the sensitivity of the model to geometric imperfections.
\end{abstract}

\section{INTRODUCTION}

Dans le domaine ferroviaire comme dans le domaine automobile, la modélisation numérique est devenue un outil indispensable pour la prévision et l'optimisation du comportement global des structures. Elle doit permettre, par des recalages intermédiaires sur des sous-ensembles, de prévoir d'une façon convenable la réalité lors d'un choc d'une rame complète. Cet article s'inscrit dans le cadre d'une tentative d'amélioration de ces recalages grâce à l'intégration des éléments simulant la soudure. Après avoir énuméré les principales caractéristiques physiques et mécaniques d'une structure mécano-soudée, nous proposons plusieurs chemins de modélisation de cette structure et ferons un bilan des différentes solutions testées.

\section{MODELISATION DES STRUCTURES FERROVIAIRES}

Depuis quelques années, la SNCF a décidé de mettre en oeuvre une politique de sécurité passive appliquée aux rames de chemin de fer, en intégrant dans les structures de caisse des zones déformables capables de dissiper les énergies mises en jeu lors d'un choc.

Sur les matériels en aluminium (remorque d'extrémité TGV Duplex), les zones d'absorption sont constituées par des plaques épaisses qui flambent suivant une direction pré-déterminée. Ces modes de déformée ainsi provoqués sont pratiquement insensibles aux tolérances et aux procédés de fabrication et leur modélisation s'en trouve grandement facilitée.

Ce n'est pas le cas des matériels en acier (motrice TGV Duplex, futur automoteur TER) où l'absorption s'effectue par flambage plastique progressif de tôles minces (épaisseur voisine de $4 \mathrm{~mm}$ ). Malgré les artifices de conception introduits dans la structure pour privilégier la déformée, l'éventail des déformations possibles reste relativement large. On cherchera donc ici à représenter numériquement la tendance majeure des déformations observées expérimentalement, afin d'affiner les prévisions futures. Cette tendance principale n'est pas toujours .obtenue avec une modélisation "simple". Si on se réfêre au tableau 1, on peut dire qu'un recalage niveau 1 est généralement atteint. Les recalages à des niveaux supérieurs sont souvent mis à mal par des efforts différents (niveau 2) et des déformées relativement êloignées de la réalité (niveau 3 : problème d'initialisation du modèle numérique, difficulté de mise en évidence du flambage généralisé, ...). Compte tenu de ces remarques, une meilleure prise en compte de la réalité physique semble nécessaire dans le cas des tôles minces.

Tableau 1 : Différents niveaux de recalage

\begin{tabular}{|c|c|c|}
\hline Niveau & \multicolumn{1}{|c|}{ Recalage } & \multicolumn{1}{c|}{ Paramètres d'influence } \\
\hline 1 & $\begin{array}{l}\text { énergétique : validité du bilan du } \\
\text { transfert énergétique }\left(\mathrm{E}_{c}, \text { Eirt, } E_{(o t)}\right.\end{array}$ & $\begin{array}{l}\text { toute dissipation énergétique : frottement. } \\
\text { phénomènes visco-élastiques, hourglass ... }\end{array}$ \\
\hline 2 & $\begin{array}{l}\text { cinématique }: \text { validité des efforts, des } \\
\text { écrasements et des temps caractéristiques }\end{array}$ & $\begin{array}{l}\text { lois de comportement (sensibilité à la vitesse,...) } \\
\text { manque de rigidité (soudures) ou souplesse (maillage) }\end{array}$ \\
\hline 3 & $\begin{array}{l}\text { géométrique }: \text { validité de la déformée } \\
\text { au cours du choc }\end{array}$ & finesse du maillage, conditions aux limites \\
\hline
\end{tabular}


En effet, la réalisation matérielle des structures absorbeuses d'énergie implique un ensemble de zaractéristiques physiques et mécaniques diagramme 1). Ces caractéristiques peuvent être zlassés en 2 grandes catégories selon qu'elles zoncernent la structure avant ou après soudage (les abréviations GEO et LC indiquent respectivement un aspect de géométrie ou de loi de comportement). Toujours dans le cas des tôles minces, on conçoit aisément que les caractéristiques issus de l'assemblage de la structure sont prépondérants par rapport à ceux précédant le soudage.

Dans une première étape d'intégration de la réalité physique, on peut tenter de rendre compte numériquement des déformations initiales de la structure avant l'écrasement dynamique. Après avoir montré l'importance des imperfections géométriques dans le cas du flambage plastique progressif, Belingardi et Gugliotta ont essayé deux méthodes basées sur une légère modification des coordonnées des noeuds du modèle [1]. Ils ont pu ainsi modéliser, avec une distribution en série de Fourier des imperfections géométriques, certains types de flambage généralisé qui n'étaient pas mis en évidence avec une structure brute ou soumise à une distribution aléatoire.

D'autres auteurs ont pris en compte l'évolution des caractéristiques mécaniques dans la soudure et la Z.A.T. (Zone Affectée Thermiquement) en modifiant la loi de comportement des éléments finis en fonction de la distance qui les sépare du cordon. Ils ont obtenu une bonne corrélation sur une structure épaisse et



sur un chassis avant de véhicule automobile [2].

En suivant toujours ce même souci de représentation de la réalité physique, il nous a paru interessant de tenter de cumuler dans une même modélisation les 2 caractéristiques évoquées ci-dessus (imperfections géométriques, lois de comportement) en prenant également en compte l'augmentation de section, et ceci grâce à l’introduction des éléments simulant la soudure.

\section{MODELISATION DES SOUDURES ET DE LEUR CARACTERISTIQUES}

Les soudures d'angle sont modélisées sous forme d'éléments finis coque reliant dans l'angle les noeuds des 2 plaques. Les soudures bout à bout sont modélisées par une modification, le long de la ligne de soudure, de l'épaisseur et de la carte matériau des éléments de la plaque. Il est à noter que les tailles des éléments "soudure" ne sont pas représentatives de la réalité, car ceci obligerait à affiner le maillage ce qui ferait chuter le pas de temps du calcul dynamique d'une façon trop importante. Plusieurs cas de calcul ont été envisagés. Ils sont résumés dans le diagramme 2 .

Les calculs crash de structures non déformées initialement sont directement effectués sous le solveur dynamique (logiciel Radioss). Deux types de conditions aux limites ont été testées (corps rigide (rigid body) ou plaque de percussion), avec ou sans distribution aléatoire des imperfections (bruit numérique d'amplitude maximale $0.1 \mathrm{~mm}$ )

En ce qui concerne les imperfections géométriques initiales de la structure, on peut noter qu'elles sont principalement dues au retrait thermique de soudage. En intégrant les contraintes résiduelles de soudage dans les éléments soudure, on peut espérer obtenir une déformée se rapprochant de la réalité. Ainsi, pour un calcul crash d'une structure déformée initialement, on procède en 2 étapes :

- intégration de la structure avec les éléments soudures dans le solveur statique (logiciel Systus). Mise sous contrainte initiale des éléments "soudure" uniquement ( $\varepsilon_{x}=\varepsilon y y=\varepsilon_{0}=-1$ (traction dans le cordon). $\varepsilon z z=\varepsilon x y=\varepsilon y z=\varepsilon z x=0$ ). Résolution. Post-traitement des résultats pour avoir un déplacement maximal d'une valeur 




Diagramme 2 : Présentation des calculs

\section{LÉgende}

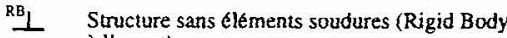
à l'avant)

1. Structure avec éléments soudures

Structure avec éléments soudures déformée après calcul statique

jo Structure avec éléments soudures déformée et contrainte après calcul statique

1 Structure sans éléments soudures déformée apres calcul statique

$\lambda^{\circ}$ Seructure sans éléments soudures déformée et contrainte après calcul statique

${ }^{R B}$ - Structure bruitée sans eléments soudures (Rigid Body à l'avant)

$\perp \quad$ Structure sans éléments soudures avec plaque avant

$\perp$ Structure bruitée sans éléments soudures avec plaque avant

de $\mathrm{x} \mathrm{mm}$. Récupération des fichiers résultat contenant soit uniquement la déformée du modèle soit la déformée et les contraintes.

- intégration de la structure déformée ou déformée et contrainte dans le solveur dynamique. Résolution avec ou sans les éléments soudures.

Dans les 2 cas, on intègre les épaisseurs et les caractéristiques mécaniques des soudures (quand elles existent) au niveau du pré-processeur dynamique. L'épaisseur des éléments "soudure" est fixée à $1 \mathrm{~mm}$ compte tenu de leur longueur et d'une section moyenne d'un joint soudé. Leur limite élastique passe de $280 \mathrm{MPa}$ (métal de base) à $340 \mathrm{MPa}$. Egalement en raison de leur taille, les éléments soudures ne font pas partie des interfaces de contact.

L'exemple traité est un élément absorbeur du chassis du futur automoteur TER (figure 1). Il contient uniquement des soudures d'angle (ces soudures sont d'ailleurs largement majoritaires dans les structures mécano-soudées). La taille des éléments du maillage est relativement grande pour permettre une introduction ultérieure de ce sous-ensemble dans le maillage du chaudron complet.

\section{RESULTATS}

\subsection{Structure non déformée par les soudures}

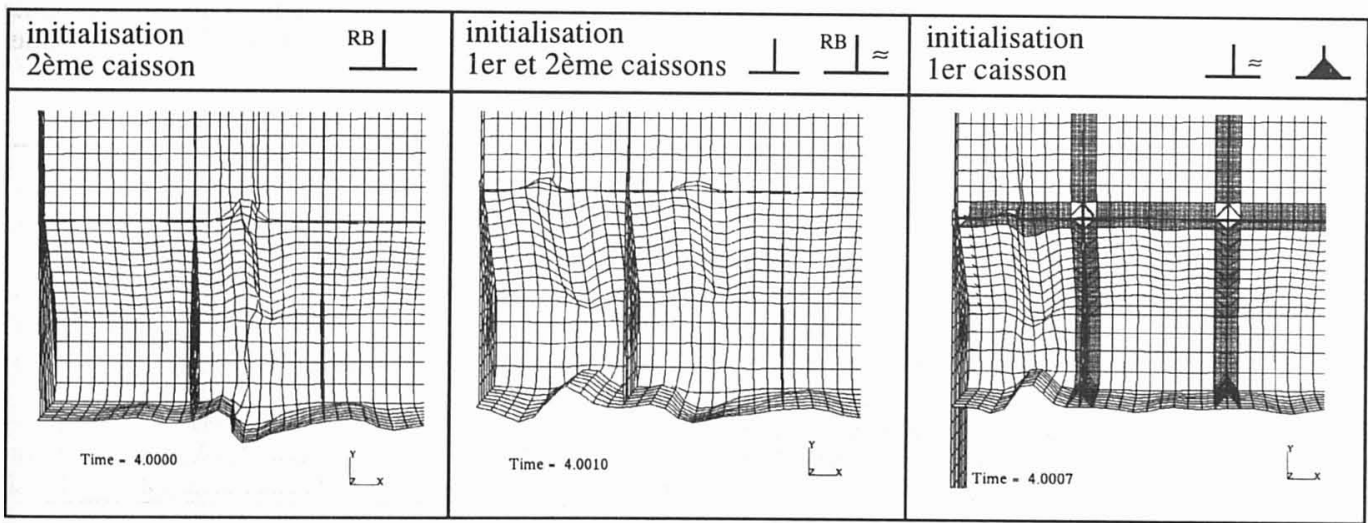

Figure 1 : Déformées numériques de la structure - Différentes conditions aux limites 
Les calculs sur les structures non déformées par les soudures mettent en évidence une sensibilité du modèle aux conditions aux limites dans les premiers instants de choc (figure 1). L'initialisation des déformées sur le deuxième caisson est fréquente sur les modèles avec corps rigide à l'avant. Le fait de le remplacer par une plaque de fermeture répercute la déformée sur les deux premiers caissons. Ce n'est qu'en rajoutant du briit que l'on obtient une initialisation correcte à l'avant pour cette structure sans rajouter les éléments "soudure".

Lorsqu'on rajoute les éléments soudures, l'initialisation se fait correctement que ce soit avec une plaque de percussion ou un Rigid Body, avec ou sans bruit numérique. L'effort d'écrasement de la structure augmente, de l'ordre de 8 à $10 \%$ par rapport à l'effort de la structure "non soudée" (figure 2). Ainsi, le fait d'intégrer les éléments soudures dans la structure la rend plus physique et du même coup moins sensible aux conditions aux limites et au bruit numérique.

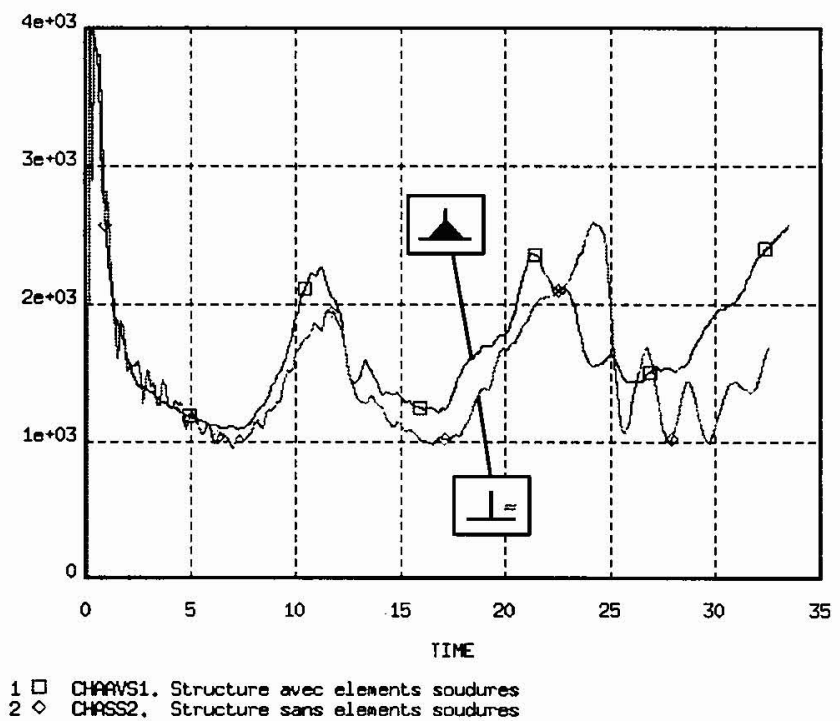

Figure 2 : Effort d'écrasement de la structure $(\mathrm{kN})$ en fonction du temps

\subsection{Structure déformée par les soudures}

La structure déformée par les soudures calculée par le solveur statique est présentée sur la figure 3. Cette structure est "post-traitée" avec un déplacement maximal de $20 \mathrm{~mm}$ pour que les déformations induites par les contraintes résiduelles de soudage soient visibles. La figure $\mathrm{Ib}$ montre, sur une coupe dans le plan $\mathrm{P}$ de cette structure, les bombés de la tôle de fermeture et le point d'inflexion dans le bas du premier caisson. Les calculs dynamiques ont eux été effectués avec deux structures déformées au maximum de $0.1 \mathrm{~mm}$ (valeur identique au bruit aléatoire) et de $1 \mathrm{~mm}$.

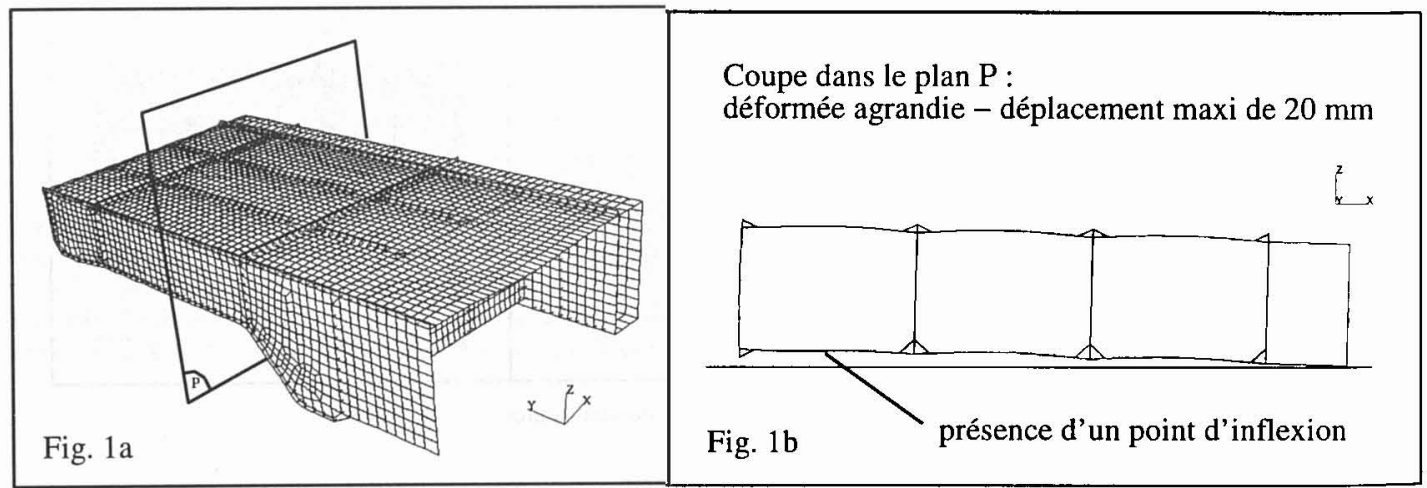

Figure 3 : Structure testée déformée après calcul statique 
Sur les structures déformées "non soudées", l'initialisation débute sur le deuxième caisson, quelque soit la valeur de l'amplitude maximale des déformées initiales $(0.1$ ou $1 \mathrm{~mm})$. Ce report est peut-être dû à la présence du point d'inflexion dans le premier caisson.

Comme pour les structures non déformées initialement, l'intégration des soudures permet à nouveau une bonne initialisation des déformées.

Dans l'ensemble, les calculs avec ou sans déformation initiale de la structure (due aux soudures) donnent les mêmes résultats en terme d'effort et de déformée sur ce type de structure.

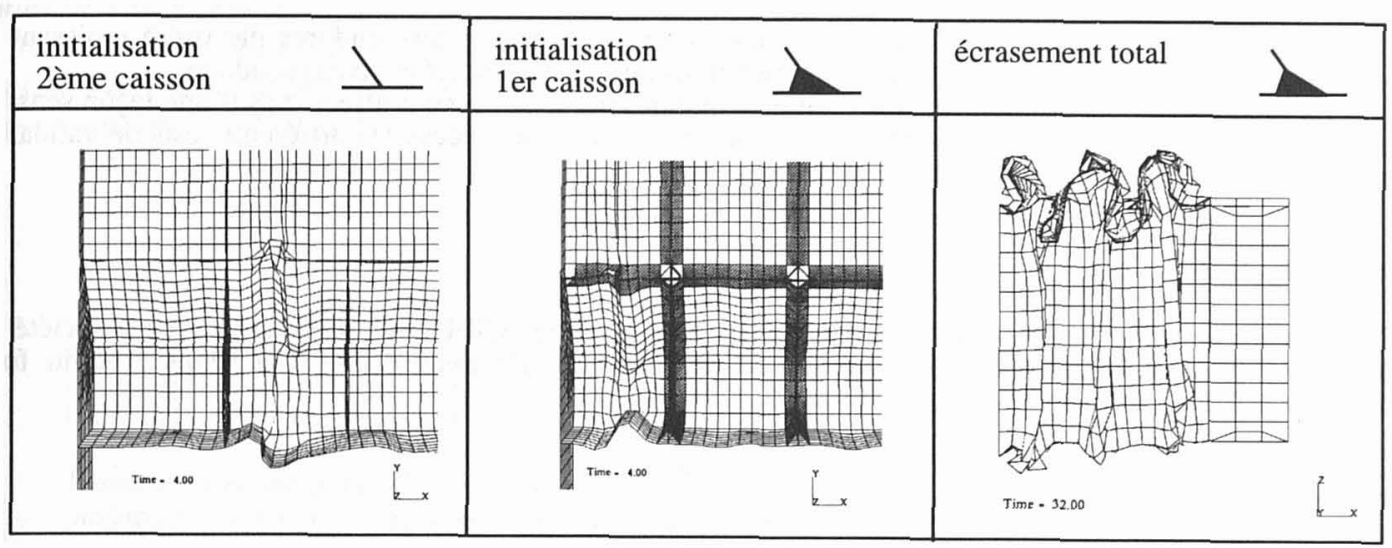

Figure 4: Déformées de la structure avec ou sans éléments soudures

\subsection{Contraintes résiduelles}

En raison des problèmes de compatibilité des fichiers résultats de contraintes, l'intégration des pré-contraintes de la structure, calculées par le solveur statique, n'a pas pu être effectuée pour l'instant dans le solveur dynamique. Cette étude est en cours actuellement.

\subsection{Sollicitations dans les soudures}

La figure 5 montre les déformations plastiques dans les différents cordons de soudure de la structure. On peut classer ces soudures en 3 catégories, par ordre croissant de sévérité de la sollicitation : soudures transversales, soudures longitudinales centrales, soudures longitudinales extérieures. On retrouve ce classement expérimentalement : les soudures transversales n'ont jamais cédé au cours des essais, les soudures longitudinales centrales rarement, les soudures longitudinales extérieures presque toujours.

Les instabilités de rupture ne sont pas prise en compte pour l'instant. En effet, les ruptures, quand elles ont lieu, se situent dans le métal de base, à environ $20 \mathrm{~mm}$ du cordon. Le problème n'est donc pas uniquement lié aux soudures. D'ailleurs, ce risque est pris en compte dès la conception : le design et le mode de fabrication de la structure sont étudiés pour minimiser les risques de rupture et les conséquences éventuelles de celles-ci $[3,4]$.

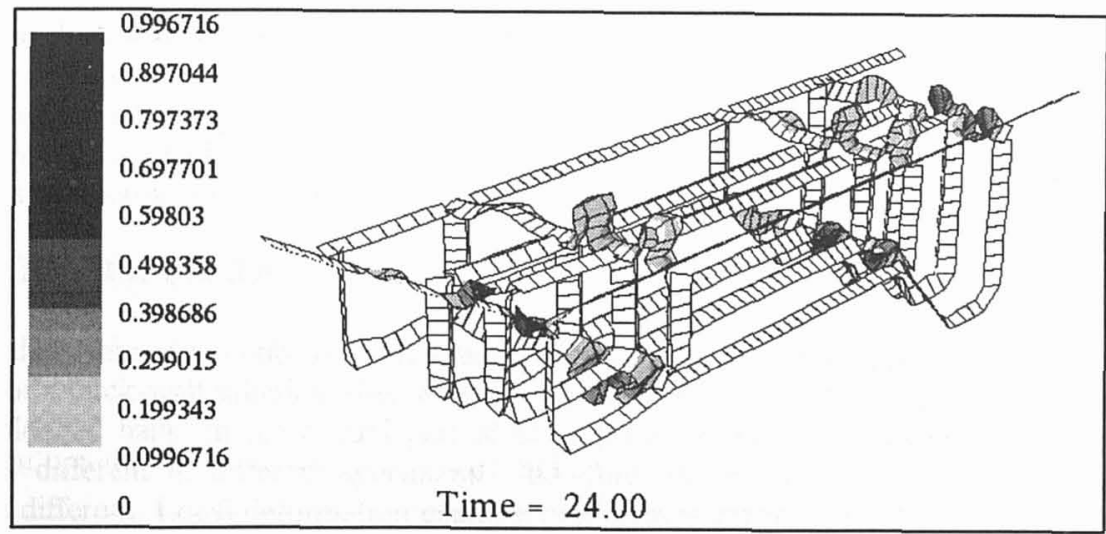

Figure 5 : Déformations plastiques dans les soudures 


\section{Conclusion}

La méthodologie présentée ci-dessus permet de se donner les degrés de liberté nécessaires à la bonne réprésentation des phénomènes physiques.

L'intégration des éléments soudures permet une initialisation correcte des déformées, quelque soit le type de conditions aux limites testées et une augmentation de l'effort d'écrasement. Ainsi complété, le modèle numérique devient plus physique et, par la même occasion, moins sensible aux paramètres: numériques et conditions initiales.

Les résultats de calculs dans les joints soudés permet de classer les soudures par ordre croissant de sollicitations. Ce critère donne une idée des préparations des bords à effectuer avant soudage.

La prise en compte des déformations initiales induites par le soudage n'affecte pas d'une façon sensible le comportement en choc de cette structure. Elle a donc passé avec succès les différents tests de validation numérique et pourra être testée expérimentalement.

\section{Remerciements}

Cette étude a été effectuée dans le cadre d'une thèse en convention CIFRE, en partenariat avec la société De Dietrich Ferroviaire, concepteur et fabricant de la remorque d'extrémité du TGV Duplex et du futur automoteur TER.

\section{Références}

[1] Belingardi G., Gugliotta A., "Recent results on crashworthiness at the Mechanical Departement of the Politeccnico of Torino", Proceedings of ICD 95.

[2] Goldhofer G., Holzner M., "Crash Simulation of Aluminium Structures with Consideration of Thermal Strength Reduction - Examples and Significance in Vehicle Development" (en allemand, in german), VDI Berichte $N^{\circ} 1283,1996$.

[3] Huss P., Dannawi M., Marya S., "Comportement des soudures bout à bout en aluminium en grandes déformations sous sollicitations dynamiques", Mécanique Industrielle et Matériaux, Vol.49, $\mathrm{N}^{\circ} 4$, Décembre 1996, pp.177-180.

[4] Huss P., Dannawi M., Marya S., "Analysis of Aluminium Butt-Welds Behaviour under Dynamic Loading", Proceedings of the 6th International Symposium, Japan Welding Society, (1996), Nagoya, pp. 305-310. 